\title{
Efeitos do ensino via CRMTS sobre leitura e construção de sentenças para crianças com autismo
}

\author{
Glenda Miranda da Paixão \\ Grauben José Alves de Assis
}

\section{RESUMO}

Crianças com diagnóstico de Transtorno do Espectro do Autismo (TEA) apresentam dificuldades na aprendizagem de leitura, fazendo-se necessário avaliar e elaborar procedimentos de ensino de repertórios de leitura para essa população. Além disso, existem poucos dados apresentados acerca do repertório de escrita. Este estudo objetivou verificar os efeitos de um treino via Constructed Response Matching to Sample - CRMTS no desenvolvimento das leituras textual e com compreensão, e da construção de sentenças, a partir de um procedimento informatizado, dividido em três estudos. Como resultado, três crianças demonstraram leitura textual e com compreensão de palavras. Duas crianças demonstraram leitura textual, compreensão e construção generalizada de sentenças de três e cinco termos. Uma criança demonstrou leitura textual, compreensão e construção generalizada de sentenças com dois termos. Houve formação de classes de equivalência e de classes ordinais. As estratégias combinadas de graduação da dificuldade da tarefa, reforçamento imediato e fornecimento de dicas, somadas ao estabelecimento do controle pelas sílabas, contribuíram para que os participantes aprendessem a ler e construir palavras e sentenças.

Palavras-chave: constructed response matching to sample; autismo; sentença; leitura.

\section{ABSTRACT \\ Effects of teaching via CRMTS on reading and sentence construction for children with autism}

Children diagnosed with Autism Spectrum Disorder (ASD) have difficulties in reading and learning, making it necessary to evaluate and elaborate procedures for teaching and reading repertoires for this population. In addition, there is little data on the writing repertoires. This study aimed to verify the effects of a training through Constructed Response Matching to Sample - CRMTS to develop textual and reading comprehension and the construction of sentences, from a computerized procedure, divided into three studies. As a result, the three children demonstrated textual reading and word comprehension. Two children demonstrated textual reading, comprehension, and generalized construction of three- and five-term sentences. One child demonstrated textual reading, comprehension, and generalized sentence construction with two terms. There was formation of equivalence classes and ordinal classes. The combined strategies of grading the difficulty of the task, immediate reinforcement and provision of tips, added to the establishment of control by syllables, contribute for participants to learn and create words and sentences.

Keywords: constructed response matching to sample; autism; sentence; reading.

O Transtorno do Espectro do Autismo (TEA), na acepção analítico comportamental de Green (2001), é uma síndrome de déficits e excessos comportamentais com base biológica, mas sensível a mudanças planejadas, entre o ambiente físico e o social. Para Spradlin e Brady (1999), as limitações que caracterizam o TEA podem ser descritas em termos de restrições do desenvolvimento do controle de estímulos, limitando o desenvolvimento de classes de

\section{Sobre os Autores}

G. M. P.

orcid.org/0000-0001-9479-2659

Universidade Federal do Pará

(UFPA) - Belém, PA

gle_miranda@hotmail.com

\section{G. J. A. A.}

orcid.org/0000-0002-6548-2544

Universidade Federal do Pará

(UFPA) - Belém, PA

ggrauben@gmail.com

\section{Direitos Autorais}

Este é um artigo de acesso aberto e pode ser reproduzido livremente, distribuído,

transmitido ou modificado, por qualquer pessoa desde que usado sem fins comerciais. 0 trabalho é disponibilizado sob a licença Creative Commons CCBY-NC.

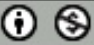




\section{H. INTERACÃO EM 4. PSICOLOGIA}

estímulos compostas por membros sem propriedades físicas similares. Essa limitação restringe 0 aprendizado de repertórios relacionados à leitura e à escrita, interligados em uma rede de relações cujos estímulos controladores fazem parte de uma classe de estímulos equivalentes (Sidman \& Tailby, 1982).

Para verificar a formação de classes de estímulos equivalentes, o procedimento de ensino por emparelhamento de acordo com o modelo (matching-to-sample - MTS) tem sido usado tradicionalmente como ferramenta nas investigações das relações condicionais entre estímulos de diferentes modalidades (auditiva, visual, tátil), arranjados experimentalmente, a partir de diferentes conjuntos de estímulos. Além disso, esse procedimento tem sido extensivamente utilizado no ensino de leitura (ver revisão de Paixão, de Souza, Kato, \& Haydu, 2013).

Entretanto, para analistas do comportamento, ler e escrever são diferentes comportamentos, a aquisição de um desses operantes não necessariamente resulta no surgimento de outro e a integração de repertórios de leitura e escrita tem sido promovida pelo procedimento denominado Constructed Response Matching to Sample - CRMTS (Reis, Postalli, \& de Souza, 2013). Este procedimento foi utilizado inicialmente no ensino de soletração, definida como a composição de uma sequência de letras em ordem específica, oralmente ou por escrito (Graham, 1983). Nas tarefas de CRMTS, a resposta correta é construída por meio da seleção de componentes individuais (de Souza \& de Rose, 2006; Dube, McDonald, Mcllvane, \& Mackay, 1991) e as escolhas podem estar relacionadas ao modelo com base nas características físicas comuns ou com base em relações arbitrárias (Paixão \& Assis, 2017). A seleção de letras em sequências específicas pode ocorrer na presença de uma palavra impressa, após a apresentação de uma palavra impressa, quando um objeto ou figura é apresentado ou, ainda quando uma palavra é ditada, ou seja, com base nas características físicas comuns ou com base em relações arbitrárias (Dube et al., 1991). Mackay e Sidman (1984) foram os pioneiros ao organizarem um procedimento de construção de palavras com um estudante com deficiência intelectual no qual modelo era composto (cartão colorido e palavra impressa referente ao nome da cor) e as comparações eram letras em cartões móveis. Dube et al. (1991), por sua vez, adotaram um procedimento informatizado, com uso de tela touchscreen, no qual, diante de um modelo visual, a criança deveria tocar nas letras apresentadas como estímulos de comparação, na sequência correta.

Stromer, Mackay, Howell, McVay e Flusser (1996) e Bagaiolo (2009) demonstraram, a partir de procedimentos envolvendo MTS e CRMTS, a formação de classes de equivalência entre figuras e palavras impressas com crianças com TEA e generalização para comportamento textual de todas as palavras, sílabas e letras utilizadas no estudo (Bagaiolo, 2009). Em outro estudo mais recente, Tanji e Noro (2011) demonstraram, a partir de tarefas de CRMTS, a construção generalizada de palavras com duas crianças, uma delas com diagnóstico de TEA.

0 procedimento por CRMTS também tem sido utilizado para o ensino de construção de sentenças. Yamamoto e Miya (1999) demonstraram que três estudantes com diagnóstico de TEA, que já liam e escreviam as palavras utilizadas no estudo, construíram, após o procedimento, 27 sentenças (de ensino e de teste) com escores de acertos de $100 \%$ para dois estudantes e $79 \%$ para um deles. Os autores utilizaram uma matriz com 27 sentenças compostas por cinco termos: sujeito, fruta, ação e duas partículas (próprias da língua japonesa). Três dessas sentenças foram utilizadas no procedimento de ensino, enquanto as outras 24 foram utilizadas nos testes. As tarefas de ensino consistiam em tentativas nas quais uma figura era apresentada como modelo, e doze palavras e quatro partículas apareciam na área de escolha, randomicamente. No pós-teste todas as 27 tentativas foram apresentadas e teste de comportamento textual também foram realizados.

A partir das evidências experimentais supracitadas, verificou-se que não há na literatura um experimento, baseado no CRMTS, que integre o ensino de construção das sílabas, pela seleção de letras, ao ensino de construção de palavras, pela seleção das sílabas ensinadas nas tentativas imediatamente anteriores. Além disso, as evidências de construção de sentenças apresentadas por Yamamoto e Miya (1999), foram encontradas apenas quando os participantes já apresentavam repertório de leitura e escrita de palavras. Dessa forma, desenvolveu-se a proposta de ensinar a discriminação de unidades textuais mínimas, seguida do ensino da discriminação de palavras inteiras, em uma mesma sequência de tentativas com objetivo de verificar o efeito desse treino no comportamento textual e na compreensão de palavras e sentenças.

Apresenta-se, assim, uma variação do procedimento de CRMTS, usualmente realizado com a seleção de letras para a construção de palavras, como descrito por Bagaiolo (2009). Além disso, o procedimento foi organizado para que o treino contemplasse apenas tentativas por CRMTS. Após a demonstração de leitura textual e com compreensão, estendeu-se o treino para a construção de sentenças, a partir da seleção de palavras, com posterior condução de testes de leitura textual, leitura com compreensão e construção de novas sentenças, avançando com relação à Yamamoto e Miya (1999), ao ser realizado com alunos com TEA que não 
apresentavam comportamento textual para palavras e sentenças antes do ingresso na pesquisa.

\section{MÉTODO}

\section{PARTICIPANTES}

Participaram três crianças (Charlie, Lucy e Sally) diagnosticadas com TEA com repertório de seguir instruções simples dadas pelo experimentador (exemplo: "aponte", "pegue") e repertório vocal para emissão de ecoicos, desconsiderando-se erros por alterações fonológicas. As idades cronológicas, as idades equivalentes obtidas a partir da aplicação do Peabody Picture Vocabulary - PPVT III (Dunn \& Dunn, 1997) e as pontuações obtidos na Childhood Autism Rating Scale - CARS (escala de 15 itens que auxilia na identificação de crianças com autismo, e diferencia o autismo leve-moderado - entre 30 e 36 pontos, do autismo grave - a partir de 37 pontos) (Pereira, 2007) estão apresentados na Tabela 1 . Os responsáveis pelos participantes foram informados sobre a pesquisa e assinaram Termo de Consentimento Livre e Esclarecido. O estudo foi aprovado pelo comitê de ética em pesquisa com seres humanos, sob parecer de no 405.206 (CEP-ICS/UFPA), de 24 de setembro de 2013.

\section{Tabela 1. Dados dos Participantes por idade cronológica, testes do PPVT e CARS.}

\begin{tabular}{lccc}
\hline Participantes & $\begin{array}{l}\text { Idade } \\
\text { cronológica } \\
\text { Anos/meses }\end{array}$ & $\begin{array}{l}\text { Idade } \\
\text { equivalente } \\
\text { (PPVT) } \\
\text { Anos/meses }\end{array}$ & $\begin{array}{l}\text { Pontuação } \\
\text { na Escala } \\
\text { CARS }\end{array}$ \\
\hline Charlie & $6 / 6$ & $5 / 4$ & 30 \\
Lucy & $11 / 10$ & $7 / 1$ & 33 \\
Sally & 8 & $1 / 9$ & 33 \\
\hline
\end{tabular}

\section{AMBIENTE EXPERIMENTAL E EQUUIPAMENTO}

A coleta de dados foi conduzida individualmente em uma sala de pesquisa de uma instituição pública de ensino e pesquisa, com relativo isolamento acústico, iluminação e climatização artificial. No procedimento informatizado utilizou-se um computador de $15^{\prime \prime}$ com tela sensível ao toque equipado com o software PROLER versão 7.1 (Assis \& Santos, 2010) para apresentação dos estímulos e registro das respostas. Foram utilizados também o programa Powerpoint e cartelas de medidas $3 \times 3,5 \mathrm{~cm}$ e $3 \times 7 \mathrm{~cm}$. Foi realizado registro audiovisual e manual (em folha de registro) das respostas dos participantes. As sessões de pré-teste e testes foram assistidas por um segundo observador, sendo o índice de concordância entre 80 a 100\%.

\section{ESTIMULOS}

Foram utilizadas, como estímulos, letras, sílabas, palavras e sentenças, escritas em fonte Calibri, tamanho 72, arquivadas em formato JPEG. Todas as palavras utilizadas eram dissílabas, do tipo Consoante-Vogal-Consoante-Vogal (CVCV), à exceção da palavra UVA. Foram utilizadas ainda figuras referentes às palavras e animações gráficas referentes aos verbos e às sentenças desenhadas, especificamente para essa pesquisa.

As figuras e animações gráficas utilizadas como consequências foram retiradas do sítio Google. Foram utilizadas, nos intervalos e ao final da sessão, brinquedos, vídeos disponíveis na internet, jogos on-line, e, para as participantes Lucy e Sally, também foram utilizados itens comestíveis.

\section{LEVANTAMENTO DE PREFERÊNCIA}

Os pais das crianças indicaram, em entrevista, os personagens, objetos, atividades lúdicas e demais itens pelos quais a criança demonstrava interesse. Esses itens foram utilizados para a seleção das animações gráficas utilizadas como consequências durante a execução das tarefas informatizadas e para a seleção dos itens do teste de preferência com múltiplos estímulos sem reposição, realizado conforme descrito por Carr, Nicholson e Higbee (2000).

\section{PROCEDIMENTO GERAL}

\section{ENSINO DAS TAREFAS DE CONSTRUÇÃO}

O participante era instruído pelo experimentador a tocar no estímulo modelo presente na parte superior da tela do computador (resposta de observação), a partir da instrução "toque", o que disponibilizava os estímulos de comparação, que poderiam ser dois, três ou cinco, dependendo do experimento em andamento, o que será pormenorizado posteriormente (as figuras e animações gráficas utilizadas como estímulos modelo foram desenhados especificamente para este experimento). Estes deveriam ser selecionados, um a um, na ordem correta, construindo um estímulo idêntico ou arbitrariamente relacionado ao modelo. Cada resposta correta era seguida por uma animação gráfica e reforço 


\section{W'INTERACÃO EM ET PSICOLOGIA}

social fornecido pela experimentadora (as animações gráficas apresentadas como consequência foram retiradas do sítio Google). Respostas diferentes da programada eram seguidas de um escurecimento da tela por aproximadamente $2 \mathrm{~s}$, e, em seguida, uma mesma configuração de estímulos era disponibilizada na tela. Para duas participantes (Lucy e Sally) foi introduzido o esquema de economia de fichas: para cada três respostas corretas era liberado acesso aos reforçadores, que poderiam ser comestíveis ou brinquedos (em média 20s) escolhidos pela participante. Todos os participantes tiveram acesso a intervalos de cinco minutos a cada 15 minutos de tarefa, no qual o participante poderia brincar com os itens disponíveis na sala, assistir vídeos ou ter acesso a jogos eletrônicos, e a sessão durava em média uma hora por dia.

A primeira tentativa de cada bloco era sempre no formato de sonda, caso a criança respondesse de acordo com o programado, o reforço era liberado. Caso não respondesse, na tentativa seguinte (idêntica à anterior) a experimentadora fornecia dica física total, na qual conduzia a mão do participante para a seleção correta. Durante as tentativas de ensino, dicas físicas parciais ou dicas verbais foram utilizadas para minimizar erros. Funcionaram como dicas físicas parciais: bloquear o movimento da mão do participante em direção ao estímulo incorreto, toque leve no antebraço do participante em direção do estímulo correto. A dica verbal era a nomeação do estímulo que o participante deveria escolher. As dicas eram retiradas gradualmente para gerar respostas independentes. A primeira dica após uma resposta diferente da programada era sempre total e, se esta fosse suficiente, nenhuma outra dica era fornecida. Porém, se na tentativa seguinte a criança ainda necessitasse de dica, esta era fornecida parcialmente, quando o participante direcionava o toque para o estímulo incorreto, ou era fornecida dica verbal se o participante não emitisse resposta por mais de $10 \mathrm{~s}$, e assim até gerar a primeira resposta independente, quando as dicas se encerravam. O critério para domínio utilizado era de três respostas corretas consecutivas independentes em cada bloco que formava a sequência. Se após nove tentativas, o critério não fosse alcançado, o bloco era encerrado e uma sequência diferente era iniciada. 0 participante poderia ser exposto até três vezes à mesma sequência e, caso não atingisse o critério de acertos, a análise de erros seria realizada.

Nos blocos cujo modelos eram sílabas, as comparações eram letras; nos blocos cujos modelos eram palavras, as comparações eram sílabas e nos blocos que tinham sentenças como modelos, as comparações eram palavras. Em todos os blocos, apenas as sílabas, letras ou palavras que compunham o modelo ficavam disponíveis.

\section{TESTE DE LEITURA TEXTUAL E LEITURA COM COMPREENSÃO}

Uma palavra/sentença impressa era apresentada como estímulo modelo e, na presença dela, era fornecida a instrução "leia", ou "o que está escrito?". Após a resposta do participante (leitura textual), ou após $3 \mathrm{~s}$ sem resposta, era solicitado que a criança tocasse no estímulo modelo, então, três figuras/animações gráficas (do tipo GIF, desenhada exclusivamente para este estudo) eram apresentadas como estímulos de comparação, sendo uma delas correspondente ao modelo. A experimentadora solicitava que a criança fizesse a seleção do estímulo de comparação com a instrução "escolha a figura" ou "qual é a figura?".

Quando o modelo era um substantivo, todas as comparações também eram substantivos, assim como quando o modelo era um verbo, todas as comparações também o eram. Cada palavra/sentença era testada uma vez. Não houve consequenciação para as escolhas do participante, e este avançava para a próxima tentativa independentemente da resposta, porém, alguns motivadores eram dispensados para manter o responder da criança: "Continue! Vamos lá!".

\section{TESTE DE CONSTRUÇÃO DE SENTENÇAS NOVAS}

Uma animação gráfica (do tipo GIF, desenhada exclusivamente para este estudo) era apresentada na tela e a experimentadora solicitava a nomeação através da instrução "o que é isso?". Após a resposta da criança ou 3s sem responder, exigia-se a resposta de observação, gerando os estímulos de comparação (palavras componentes da sentença). Independentemente da resposta de nomeação, era solicitado à criança que escrevesse a sentença através da instrução "escreva". Cada palavra/sentença era testada apenas uma vez. Assim como no teste anterior, não houve consequenciação para as escolhas do participante, este avançava para a próxima tentativa independente do responder e eram dispensados alguns motivadores. A seguir serão pormenorizados os procedimentos de cada estudo.

\section{ESTUD0 1}

Neste estudo verificou-se o efeito de um procedimento de ensino sobre a leitura textual e com compreensão de doze palavras que compõem as sentenças utilizadas neste estudo e no Estudo 2. 0 procedimento baseou-se na combinação de construção de sílabas e palavras na mesma sequência. Os estímulos foram de duas modalidades: visual e auditiva. $\mathrm{Na}$ modalidade visual havia letras, sílabas e palavras impressas (SAPO, VACA, TATU, GATA, BOLA, SINO, PULA, COME, CAVA, ROLA, GIRA, TOCA), figuras e animações gráficas. $\mathrm{Na}$ 


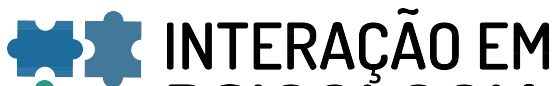 PSICOLOGIA}

modalidade auditiva estavam as sílabas e palavras ditadas.

\section{PROCEDIMENTO}

\section{ETAPA 1 - PRÉ-TESTE}

Foi realizado um pré-teste de leitura textual das palavras do estudo. Cada palavra foi apresentada três vezes, totalizando 36 tentativas, solicitando-se que o participante as lesse em voz alta.

\section{ETAPA 2- ENSINO POR CRMTS DE PALAVRAS}

Cada palavra foi ensinada conforme descrito no procedimento geral, em uma sequência contendo sete blocos de construção: 1. primeira sílaba sob controle de uma sílaba impressa; 2. primeira sílaba sob controle de uma sílaba ditada; 3. segunda sílaba sob controle de uma sílaba impressa; 4. segunda sílaba sob controle de uma sílaba ditada; 5. palavra sob controle da palavra impressa; 6 . palavra sob controle da palavra ditada; 7. palavra sob controle de uma figura. 0 participante só iniciava um bloco após atingir critério de acertos no bloco anterior. Esta sequência visou integrar o ensino de discriminação de sílabas e o ensino de discriminação de palavras, reportados de forma isolada na literatura da Análise do Comportamento, bem como minimizar a ocorrência do controle restrito, uma vez que buscava estabelecer o controle pela primeira sílaba, em seguida, pela segunda sílaba e, finalmente pela palavra por elas compostas. Após alcançar o critério de três respostas independentes em cada bloco da sequência de uma palavra, o ensino de outra palavra era iniciado. Após atingir o critério de acertos nas sequências de todas as palavras ensinadas, o teste de leitura textual e com compreensão de palavras era iniciado, conforme descrito a seguir.

Procedimentos adicionais foram aplicados à participante Lucy, utilizando-se o software PROLER $®$ e o PowerPoint para ensino de discriminação entre as palavras TOCA e ROLA. Os procedimentos foram: 1. MTS por identidade das palavras TOCA e ROLA com consoantes em destaque e um ou dois estímulos de comparação; 2. MTS por identidade das palavras TOCA e ROLA, sem destaque das consoantes e três estímulos de comparação; 3 . retorno às tarefas de construção das palavras TOCA e ROLA.

\section{ETAPA 3 - TESTE DE LEITURA TEXTUAL E LEITURA COM COMPREENSÃOO DAS PALAVRAS}

Os testes foram realizados conforme descrito no procedimento geral. Quando o participante apresentou a leitura de todas as palavras, foi encaminhado ao Estudo 2

\section{RESULTADOS}

Durante o pré-teste, as três crianças não apresentaram leitura das palavras utilizadas neste estudo. Nos blocos de construção das sílabas, os três participantes necessitaram de uma ou duas exposições para atingir o critério de acertos na presença dos modelos impressos e ditados. Apesar de ter atingido critério de aprendizagem, observou-se que a participante Lucy necessitou de um número de tentativas variado, chegando a oito tentativas diante de modelos auditivos, e apresentou necessidade de dicas verbais e de dicas físicas parciais. Dessa forma, optou-se por fazer um procedimento de ensino de leitura receptiva das sílabas, na qual, diante do som emitido pela experimentadora, a participante deveria selecionar uma sílaba impressa em meio a um conjunto de sílabas apresentadas em cartelas. Lucy necessitou de cinco exposições até apresentar leitura receptiva das dezoito sílabas utilizadas no estudo. Foi conduzido, ainda, um treino de leitura textual, no qual a criança deveria nomear sílabas apresentadas em cartelas. Caso, diante da sílaba impressa apresentada, a participante nomeasse corretamente, reforço social era liberado, caso não nomeasse, a experimentadora disponibilizava o modelo oral e solicitava o ecoico. Lucy necessitou de seis exposições ao procedimento de ensino para apresentar leitura textual das dezoito sílabas. Apenas após apresentar repertório de leitura receptiva e leitura textual de sílabas, a participante foi conduzida ao ensino de construção de palavras.

Todos os participantes necessitaram de uma a três exposições para atingir o critério de acertos nos blocos de construção da palavra sob controle de modelos visuais impressos, modelos auditivos e figuras.

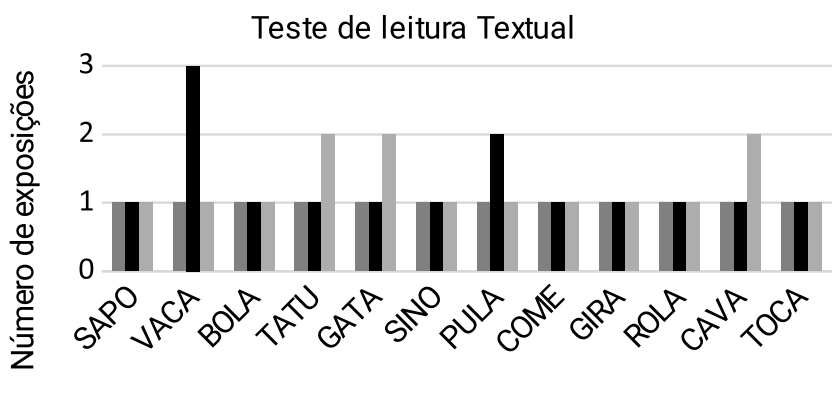

Palavras impressas

$$
\text { - Charlie } \square \text { Lucy } \square \text { Sally }
$$

Figura 1 . Quantidade de exposições aos testes de leitura textual por palavra.

Com relação aos testes de leitura textual de palavras (Figura 1), Charlie apresentou, na primeira exposição, leitura 


\section{W'INTERACÃO EM ET PSICOLOGIA}

textual das palavras. Lucy apresentou prontamente leitura textual para dez palavras e Sally necessitou de apenas uma exposição para nove palavras. Lucy e Sally apresentaram evidências de controle restrito por uma sílaba e também pela primeira letra. Lucy, diante de VACA, nomeou "cava". Verificouse dessa forma a possibilidade de controle restrito pela unidade "CA", e diante da palavra PULA, nomeou "pu" e "u" em sequência, evidenciando controle apenas por parte dos estímulos apresentados. Sally, diante da palavra GATA, nomeou "gira", diante de TATU, nomeou "tota" (equivalente à "toca", pois, a criança apresenta dificuldades fonológicas) e diante de CAVA nomeou "come", evidenciando controle pela primeira letra.

Desse modo, Lucy foi reexposta as tentativas da palavra VACA e à Sally foram ensinadas, aos pares, as discriminações entre as palavras GATA e GIRA, CAVA e COME, TATU e TOCA, tendo ambas as crianças demonstrado aprendizado das palavras na segunda ou terceira exposição.

Com relação à leitura com compreensão (Figura 2), Charlie apresentou o repertório na primeira exposição a todas as palavras. Sally precisou de duas exposições a SAPO e SINO, apresentando leitura com compreensão para as dez outras na primeira exposição. Lucy apresentou leitura com compreensão prontamente para dez palavras, porém respondia aleatoriamente a TOCA e ROLA. Após a aplicação dos procedimentos adicionais já descritos, realizou leitura com compreensão das duas palavras.

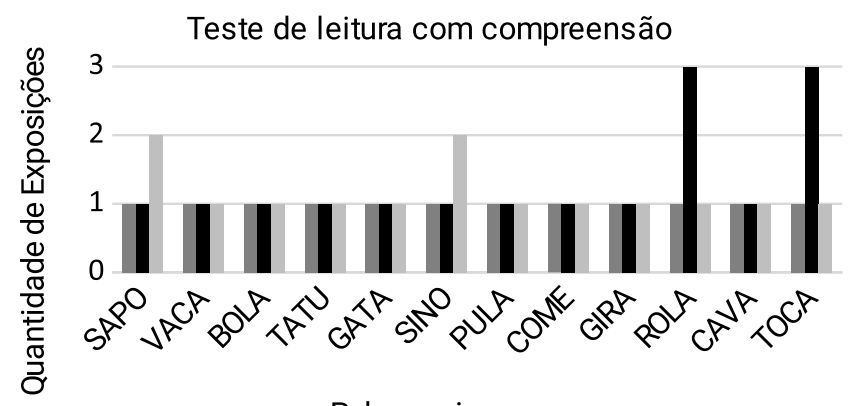

Palavras impressas

$$
\text { - Charlie — Lucy } \text { Sally }
$$

Figura2 . Quantidade de exposições aos testes de leitura com compreensãopor palavra.

\section{DISCUSSÃO}

A participante Lucy necessitou de quantidade maior de tentativas para alcançar critério de acertos independentes. Os dados de Dube et al. (1991) também mostraram uma variabilidade comportamental durante as etapas de treino. Para um dos participantes, os percentuais de respostas corretas durante o treino variaram entre 47 e $99 \%$. Bagaiolo (2009) demostrou que a criança com TEA necessitou de um número maior de tentativas quando utilizou os mesmos procedimentos adotados com crianças típicas.

Charlie, Lucy e Sally demonstraram repertório de leitura textual e de leitura com compreensão, corroborando Mackay (1985), Mackay e Sidman (1984) e Dube et al. (1991), que documentaram formação de classes de equivalência a partir do procedimento de CRMTS. Segundo Mackay e Sidman (1984), os procedimentos de MTS e CRMTS fornecem suporte para estabelecer a compreensão de formas simples de linguagem textual e falada, sendo úteis com pessoas cujas habilidades de linguagem receptiva e produtiva são deficientes, como as crianças com diagnóstico de TEA são geralmente descritas.

Foram observadas, conforme descrito na seção de resultados, evidências de controle restrito de estímulos (da Hora \& Benvenuti, 2007; Dube \& Mcllvane, 1999), pois a similaridade física entre as palavras TOCA e ROLA (consoante- vogal $O$ - consoante- vogal A), e as primeiras letras iguais nas palavras SINO e SAPO, GATA e GIRA, e TATU e TOCA, podem explicar as respostas emitidas por Lucy e Sally na primeira exposição ao teste.

\section{ESTUD0 2}

O objetivo deste estudo foi verificar o efeito de um ensino de construção de sentenças, a partir da seleção de palavras, na leitura textual e com compreensão delas e na construção de sentenças novas. Foram ensinadas seis sentenças, os estímulos utilizados foram de duas modalidades: visual e auditiva. Na modalidade visual, havia palavras impressas (ver estudo 1), figuras e animações gráficas e na auditiva estavam as sentenças ditadas (O SAPO PULA, A VACA COME, O TATU CAVA, A BOLA GIRA, O SINO TOCA, A GATA ROLA).

\section{PROCEDIMENTO}

\section{ETAPA 1 - ENSINO POR CRMTS DAS SENTENÇAS}

O ensino de cada sentença foi realizado em uma sequência de três tipos de bloco: 1 . construção da sentença sob controle de uma sentença impressa, havendo esvanecimento1 do modelo, 2. construção da sentença sob controle de uma sentença ditada e, 3. construção da sentença sob controle de uma animação gráfica.

O esvanecimento foi introduzido após apresentação de três acertos consecutivos na construção da sentença sob controle de um modelo impresso, retirando-se cada palavra do estímulo modelo da direita para a esquerda, um único 


\section{MI* INTERACÃO EM LF PSICOLOGIA}

acerto era exigido em cada um dos blocos de esvanecimento. Após a tentativa na qual apenas o artigo era apresentado como modelo, o bloco de tentativas de construção sob controle de um modelo auditivo era iniciado e, em seguida, o bloco de tentativas de construção sob controle de uma animação gráfica. Após o critério de acertos de três respostas corretas consecutivas em cada bloco de uma sentença ser alcançado, uma sequência de blocos de uma nova sentença era iniciado. Quando o critério de acertos foi alcançado para todas as sentenças ensinadas, os testes de leitura textual e com compreensão foram realizados.

Para a participante Lucy, procedimentos adicionais foram utilizados. Os procedimentos adicionais 1 e 2 envolveram a seleção do artigo correto para as sentenças (sujeito + verbo), o procedimento adicional 3 envolvia a seleção de um artigo diante de uma sentença, no procedimento adicional 4 a participante deveria selecionar a sentença escrita com o artigo correto e o procedimento adicional 5 envolvia a construção da sentença, com mudança gradual da cor do artigo presente no estímulo modelo.

\section{ETAPA 2 - TESTE DE LEITURA TEXTUAL E LEITURA COM COMPREENSÃO DE SENTENÇAS}

Os testes foram aplicados conforme descrito no procedimento geral. 0 teste de construção de sentenças novas foi realizado para duas participantes.

\section{ETAPA 3 - TESTE DE CONSTRUÇÃO DE SENTENÇAS NOVAS}

Na presença de novas animações gráficas, o participante era solicitado a construir sentenças novas (O SAPO COME, A VACA TOCA, A BOLA PULA, O SINO GIRA, O TATU ROLA e A GATA CAVA) formadas pelas palavras que estavam presentes nas sentenças de ensino, conforme descrito no procedimento geral.

\section{RESULTADOS}

Charlie e Sally necessitaram de apenas uma exposição a cada bloco de ensino para atingir o critério de acertos, exceto para o bloco de O SAPO PULA, cujo modelo era a sentença impressa, para o qual Sally necessitou de duas exposições. Entretanto, esse foi o primeiro bloco a ser apresentado à criança.

Iniciou-se o treino de Lucy com a sentença O SAPO PULA, porém, após quatro exposições, a criança não atingiu o critério de acertos. Passou-se, então, para o ensino de outra sentença, porém, o critério de acertos também não foi atingido. Observou-se que a maior parte dos erros apresentados foi a não seleção do artigo. Os procedimentos adicionais descritos na sessão de método foram conduzidos, e, mais uma vez, o critério de acertos nas respostas independentes não foi atingido. Dessa forma, optou-se por conduzir o Estudo 2 removendo-se o artigo, devendo a criança construir as sentenças de apenas dois termos (sujeito e verbo). Assim, necessitou-se de apenas uma exposição para cada bloco cujo modelo era a sentença impressa, e de duas exposições para os blocos cujos modelos eram auditivos nas sentenças TATU CAVA e GATA ROLA. Nas sentenças nas quais uma animação gráfica era o modelo, necessitou de duas exposições para as sentenças VACA COME E SINO TOCA.

Os testes de leitura textual e com compreensão foram então conduzidos. Charlie e Lucy necessitaram de apenas uma exposição para cada sentença, Sally precisou de duas exposições no teste de leitura textual da sentença A BOLA GIRA e nos testes de leitura textual e com compreensão da sentença O SINO TOCA. Nos testes de construção de sentenças novas, Charlie e Sally necessitaram de duas exposições apenas para a sentença A GATA CAVA, Lucy necessitou de duas exposições à sentença SINO GIRA. As demais sentenças foram construídas corretamente já na primeira exposição.

\section{DISCUSSÃO}

Os três participantes demonstraram leitura textual, leitura com compreensão e produção de sentenças novas. Corroborando Bandeira, Assis e Souza (2016), a formação de classes de estímulos equivalentes parece ter sido demonstrada, a partir do procedimento de CRMTS, havendo três possíveis classes formadas: artigos, sujeitos e verbos, ou: primeiros, segundos e terceiros. Entretanto, ao ser analisado o procedimento, considera-se importante, em estudos futuros, testes adicionais envolvendo as categorias formadas. Um exemplo disso seria um teste no qual o participante, na presença de um verbo como modelo, devesse selecionar um verbo, na presença de verbos e substantivos como comparações; ou diante de um substantivo como modelo, selecionar um outro substantivo na presença de substantivos e verbos como comparações, e assim por diante.

Para Lucy, somente as classes de sujeito e verbo, ou primeiros e segundos parecem ter sido formadas. Mackay e Fields (2009) propõem que a posição dos eventos em sequência é uma variável crítica para aprendizagem da leitura com compreensão e da escrita de sentenças. Os autores sugerem que a partir do ensino de duas ou mais sentenças, é possível verificar que os estímulos que ocuparam a mesma posição passam a funcionar como membros das classes equivalentes de primeiros, segundos, terceiros, os estímulos 
passam a ser intercambiáveis e substituíveis e os diferentes nomes próprios, os verbos, os substantivos, compõem novas sentenças não diretamente treinadas.

O esvanecimento parece ter sido importante para a transferência de controle de estímulo da construção da sentença diante do modelo impresso para o modelo ditado, corroborando os dados de Assis, Fonseca e Bandeira (2014) que utilizaram esta mesma ferramenta metodológica para ensinar leitura e construção de sentenças para crianças em idade escolar que já apresentavam leitura de palavras.

Neste estudo, Lucy também precisou de procedimentos adicionais. Bagaiolo (2009) realizou sete procedimentos adicionais para a manutenção do responder do seu participante, permitindo a continuidade dele na pesquisa. Entretanto, após cinco procedimentos adicionais, avaliou-se que a participante não aumentava a frequência do responder diante do artigo, permanecendo esse comportamento com grande variabilidade. Para minimizar a história de erros e para diminuir a frequência de dicas fornecidas, optou-se por retirar o artigo, utilizando sentenças de apenas dois componentes. Essa nova configuração permitiu a continuidade da criança na pesquisa, apresentando evidências de formação de classes de equivalência.

\section{ESTUD0 3}

O objetivo desse estudo foi verificar a expansão do Estudo 2, com a ampliação da quantidade de componentes de uma sentença, além da inserção de novas palavras e sentenças. Realizou-se também a verificação da manutenção do repertório em testes adicionais de manutenção. Foram utilizados estímulos nas modalidades visual e auditiva. Dentro da modalidade visual, havia sílabas e palavras impressas, figuras e animações gráficas. Na modalidade auditiva estavam as palavras e as sentenças ditadas.

\section{PROCEDIMENTO}

\section{ETAPA 1 - PRÉ-TESTE DE LEITURA TEXTUAL DAS PALAVRAS}

As palavras foram apresentadas em PowerPoint. $\mathrm{Na}$ presença da palavra, a experimentadora fornecia a instrução "Leia", após a resposta da criança ou após 3s sem responder, uma nova palavra era disponibilizada na tela.

\section{ETAPA 2 - PROCEDIMENTO DE CRMTS DE PALAVRAS}

Nessa fase, foram apresentadas as palavras (GUTO, RITA, NINA, TOMA, PEDE, COME, BALA, BOLO, SUCO, UVA, CAJU, coco) da mesma forma descrita no Estudo 1, excetuando-se os blocos de construção das sílabas. Após atingir o critério de acertos em cada bloco de ensino para todas as palavras, o teste de leitura textual e com compreensão eram aplicados.

\section{ETAPA 3 - TESTE DE LEITURA TEXTUAL E DE LEITURA COM COMPREENSÃO DAS PALAVRAS}

O teste de leitura textual e de leitura com compreensão das palavras era executado conforme descrito no procedimento geral.

\section{ETAPA 4 - PROCEDIMENTO DE CRMTS DE SENTENÇAS COM TRÊS E CINCO TERMOS}

Para duas participantes (Lucy e Sally) inicialmente foram ensinados dois conjuntos de sentenças, sendo o conjunto 1 composto pelas sentenças: GUTO TOMA SUCO, NINA COME BOLO e RITA PEDE BALA, e o conjunto 2 composto pelas sentenças: SUCO DE CAJU, BOLO DE COCO e BALA DE UVA, na presença de modelos visuais (sentença impressa) e auditivos (sentença ditada). Após três acertos consecutivos para cada sentença, na presença de cada modelo, iniciou-se o ensino das sentenças com cinco termos (Charlie foi exposto diretamente ao ensino nessa configuração), sendo elas GUTO TOMA SUCO DE CAJU, NINA COME BOLO DE COCO e RITA PEDE BALA DE UVA. O ensino foi realizado utilizando-se o mesmo procedimento descrito no Estudo 2.

\section{ETAPA 5 - TESTE DE LEITURA TEXTUAL E DE LEITURA COM COMPREENSÃOO DAS SENTENÇAS}

Este teste foi conduzido nas mesmas condições descritas no procedimento geral.

\section{ETAPA 6 - TESTES DE CONSTRUÇÃO DE SENTENÇAS NOVAS}

Teste realizado segundo descrito no procedimento geral. As sentenças recombinadas foram: RITA PEDE BALA DE COCO; GUTO TOMA SUCO DE UVA; NINA COME BOLO DE CAJU; RITA COME BOLO DE UVA; GUTO PEDE BALA DE CAJU e NINA TOMA SUCO DE COCO.

\section{ETAPA 7 - TESTES DE MANUTENÇÃO DO DESEMPENHO [FOLLOW UP]}

Após períodos de 15 e 30 dias sem contato com as contingências de ensino e testes, os participantes que apresentaram construção de sentenças novas no Estudo 3 foram reexpostos a esse teste para verificação da manutenção do desempenho. 


\section{H NTERAC̄̈OEM ET PSICOLOGIA}

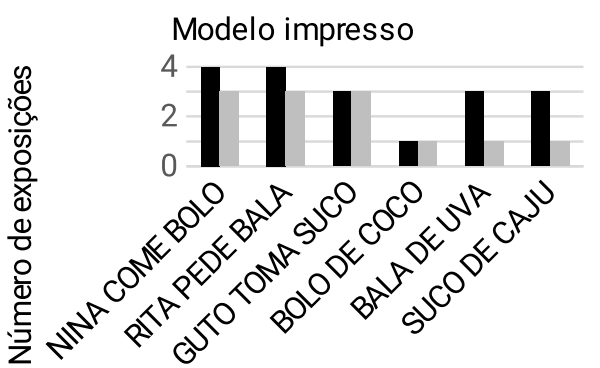

Sentença impressa

- Lucy $\backsim$ Sally

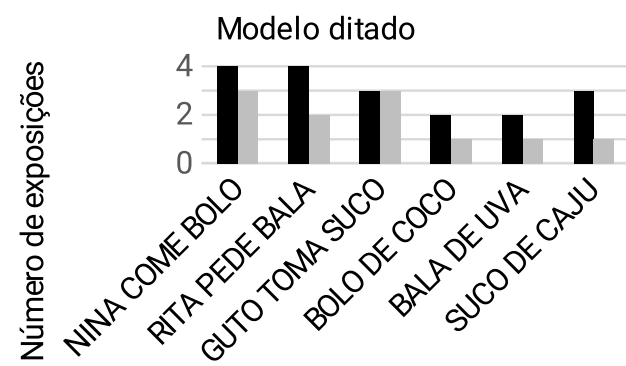

Sentença ditada

Figura3 . Quantidade de exposições aos blocos de ensino das sentenças sob modelos impressos e ditados.

\section{RESULTADOS}

No Estudo 3, as três crianças não apresentaram leitura textual das palavras no pré-teste como resultado dos estudos anteriores. Charlie apresentou leitura de seis palavras, porém sem fluência, dessa forma, foram ensinadas as doze palavras, com necessidade de uma a duas exposições para cada participante. Em seguida, Lucy e Sally foram expostas ao ensino de sentenças de três termos. Lucy necessitou de uma exposição para a sentença BOLO DE COCO, sob modelo visual, porém necessitou de três a quatro exposições para as demais cinco sentenças. Sally precisou de uma a três exposições para o ensino de cada sentença em cada tipo de bloco (ver Figura 3).

Iniciou-se, então, o ensino das sentenças com cinco termos. Charlie necessitou de apenas uma exposição, Sally necessitou de duas exposições para a construção de uma sentença, em todos os blocos, e de uma exposição para as demais sentenças em todos os blocos. Após quatro exposições aos blocos de ensino de cada sentença, Lucy não atingiu o critério e apresentava grande variabilidade comportamental. Este dado conduziu ao encerramento da participação de Lucy na pesquisa.

Os testes de leitura textual e com compreensão das três sentenças foram conduzidos para Charlie e Sally. Ambos apresentaram os dois tipos de leitura na primeira exposição. No teste de construção de sentenças novas, Charlie construiu corretamente quatro sentenças novas e Sally construiu cinco na primeira exposição ao teste. Nos dois testes de manutenção (quinze e trinta dias após a aplicação dos testes), Charlie obteve $100 \%$ de acertos, Sally construiu corretamente cinco das seis sentenças testadas.

\section{DISCUSSÃO}

0 estudo 3 permitiu verificar a extensão dos repertórios aprendidos para sentenças mais longas, apresentando componentes novos: a partícula "de" (preposição) e o complemento. Yamamoto e Miya (1999), Assis et al. (2014) e Bandeira et al. (2016) também utilizaram sentenças de cinco termos, porém os dois primeiros tiveram participantes que já liam palavras anteriormente à introdução dos mesmos nos estudos apresentados, sendo uma das proposições de Yamamoto e Miya (1999) a realização com crianças autistas que não apresentassem repertório de leitura de palavras.

Uma diferença do procedimento torna-se relevante: o ensino de sentenças aqui apresentado tinha como parte da sequência de ensino blocos com o procedimento de esvanecimento do tipo fading out, assim como no estudo de Assis et al. (2014). No estudo de Bandeira et al. (2016) o procedimento de fading in foi utilizado apenas como procedimento de correção, porém, quatro dos sete participantes alcançaram critério somente após a introdução deste procedimento (fading in), sugerindo que, para o ensino de sentenças com muitos elementos, é necessário tornar o aprendizado gradual.

Com relação aos testes de manutenção, as respostas das crianças participantes do estudo de Bandeira et al. (2016) apresentaram escores entre 80 e 100\%, tendo sido registrado para quatro crianças um decréscimo com relação aos testes imediatamente anteriores, e para três crianças a manutenção ou o aumento da frequência de respostas corretas. As três que apresentaram manutenção do desempenho foram expostas ao procedimento de fading in, o que, mais uma vez, evidencia a importância do ensino gradual, justificando a escolha do procedimento apresentado na sessão de método, permitindo a Charlie e Sally a manutenção do repertório após 15 e 30 dias.

\section{DISCUSSÃO GERAL}

Os estudos de Yamamoto e Miya (1999) e de Bagaiolo (2009) demonstraram a formação de classes de equivalência 


\section{L"INTERACÃO EM ET PSICOLOGIA}

em indivíduos com diagnóstico de TEA, corroborando os dados aqui obtidos. Entretanto, além desta classe, pode-se afirmar que houve a formação de classes gramaticais de sujeitos, verbos e complementos, demonstrando um repertório verbal mais extenso e complexo, e a formação de classes ordinais, pois os estímulos que ocupavam uma determinada posição em uma sequência quando intercalados com estímulos de outras sequências, foram selecionados na mesma posição que ocuparam durante o treino, corroborando Mackay e Fields (2009).

Os resultados indicando formação de classes de equivalência e classes ordinais apresentados nos estudos $2 \mathrm{e}$ 3, principalmente no Estudo 3 após a ampliação dos componentes das sentenças, podem ser atribuídos às características do procedimento geral adotado, pois este permitiu graduar a dificuldade da tarefa, reforçamento imediato e fornecimento de dicas, retiradas gradualmente, até o responder independente, além de ser informatizado. Testes adicionais de verificação das classes, conforme descrito na discussão do estudo 2, poderiam ser incluídos em estudos vindouros. Dube et al. (1991), Stromer e Mackay (1992) e Yamamoto e Miya (1999) demonstraram a importância de pesquisas que utilizam procedimentos de ensino informatizados, pois estes exigem topografias de respostas motoras mais simples (neste caso, toque na tela), e ainda, possibilitam feedback imediato (acertos e erros).

Outra variável que pode ter contribuído para esses resultados foi o estabelecimento do controle pelas sílabas. Estudos que estabelecem inicialmente o controle pelas sílabas têm apresentado menor variabilidade intersujeitos e aumento nos níveis de acerto para leitura de palavras (Hanna, Karino, Araujo, \& de Souza, 2010). Entretanto, o controle por unidades mínimas não impediu dados que evidenciassem o controle restrito de estímulos, o que tem sido um problema frequente no ensino a indivíduos com TEA. Estudos têm sido conduzidos no sentido de identificar e reduzir a ocorrência do controle restrito (Alves, Assis, Kato, \& Brino, 2011; da Hora \& Benvenutti, 2007; Dube \& Mcllvane, 1999; Walpole, Roscoe \& Dube, 2007). Neste estudo, optou-se por fortalecer as relações entre as palavras ditadas e as palavras impressas, melhorar o controle por todas as letras que compunham as palavras, dando destaque às letras que as diferenciavam e ainda reexpondo o participante à construção das palavras para as quais erros por provável controle restrito tivessem ocorrido.

O uso da dica física e de dicas parciais parece ter sido um favorecedor do aprendizado, induzindo ao controle por seleção e, portanto, diminuindo a ocorrência de erros. Perez e Tomanari (2014) realizaram um levantamento acerca dos procedimentos de indução do controle por seleção e rejeição e tal levantamento sugere que a dica do S+ tem permitido que o controle discriminativo seja estabelecido de modo eficaz, em especial para participantes com desenvolvimento atípico.

Para Lucy e Sally, a introdução da economia de fichas foi necessária para reduzir os comportamentos de fuga observados principalmente diante da repetitividade da tarefa, mesmo com a ocorrência de intervalos. A economia de fichas tem sido uma das tecnologias mais importantes de modificadores de comportamento aplicada por analistas do comportamento nos últimos 40 anos e tem demonstrado sucesso em diferentes estudos comportamentais sobre TEA. As fichas funcionam como estímulos reforçadores secundários que adquirem o seu valor de reforço por meio do emparelhamento com outros reforçadores. A manutenção do engajamento de Sally e Lucy nas tarefas após a introdução do sistema de economia de fichas encontra suporte na literatura (Shogren, Machalicek, Rispoli, \& O'Reilly, 2011), que tem demonstrado um aumento no engajamento em tarefas acadêmicas de leitura.

Essas estratégias combinadas contribuíram para que os participantes aprendessem a ler e construir palavras e sentenças. Lucy não demonstrou repertório de construção de sentenças com cinco elementos, porém, deve-se considerar que esta participante era a de maior idade, já tendo sido exposta a outras tentativas de alfabetização no ambiente escolar sem sucesso, e essa história pré-experimental culminou com reforçamento de comportamentos concorrentes com o aprendizado de leitura e escrita nos moldes desta pesquisa, como soletração (nomeação de letras diante de palavras) e dependência de dicas. Entretanto, Lucy apresentou um aumento de repertório de leitura e escrita quando se compara o repertório final e o inicial da participante neste estudo, uma vez que não apresentou leitura textual de palavras no pré-teste, porém apresentou, ao final do estudo, leitura textual e com compreensão de 23 palavras e seis sentenças com dois termos, bem como escreveu sentenças novas compostas por dois termos e por três termos.

Sugere-se que variáveis que minimizem ou corrijam o controle restrito sejam melhor investigadas. Além disso, devese verificar o efeito de procedimentos de ensino com a utilização de palavras com três ou mais sílabas ou, por exemplo, com encontros vocálicos ou consonantais para assegura a eficiência do procedimento.

\section{CONTRIBUIÇÃO DE CADA AUTOR}

G.M.P.: conceitualização, obtenção de financiamento, investigação e visualização, redação - rascunho; G.M.P. e G.J.A.A.: supervisão, redação - revisão e edição. 


\section{W INERACĀOEM ET. PSICOLOGIA}

\section{DECLARAÇÃO DE CONFLITOS DE INTERESSES}

Os autores declaram que não há conflitos de interesses no presente artigo.

\section{DECLARAÇÃO DE FINANCIAMENTO}

A pesquisa relatada no manuscrito foi financiada parcialmente pela bolsa de doutorado da primeira autora (Conselho Nacional de Desenvolvimento Científico e Tecnológico - CNPQ, Processo: 141643/20137)

\section{REFERÊNCIAS}

Alves, K. R. S., Assis, G. J. A., Kato, O. M., \& Brino, A. L. F. (2011). Leitura recombinativa após procedimentos de fading in de sílabas das palavras de ensino em pessoas com atraso no desenvolvimento cognitivo. Acta Comportamentalia, 19, 183-203.

Assis, G. J. A., \& Santos, M. B. (2010). PROLER (Sistema Computadorizado de Ensino de Comportamentos Conceituais). Belém: Universidade Federal do Pará.

Assis, G. J. A., Fonseca, A. C. G., \& Bandeira, T. M. (2014). Efeito do ensino da resposta por construção de sentenças sobre a leitura generalizada recombinativa. Comportamento em Foco, 4, 155-171.

Bagaiolo, L. (2009). Padrões de Aquisição de Discriminação Condicional durante a Emergência do Controle por Unidades Verbais Mínimas na Leitura em Crianças com Autismo e Desenvolvimento Típico. (Tese de doutorado não publicada), Universidade de São Paulo, São Paulo SP.

Bandeira, T. M., Assis, G. J. A., \& Souza, C. B. A. (2016). Efeito do ensino por resposta construída simultânea e com atraso sobre a produção e leitura de sentenças. Perspectivas em Análise do Comportamento, 7, 165-182. http://dx.doi.org/10.18761/pac.2016.012

Carr, J. E., Nicholson, A. C., \& Higbee, T. S. (2000). Evaluation of a brief multiple-stimulus preference assessment in a naturalistic context. Journal of Applied Behavior Analysis, 33, 353-357. http://dx.doi.org/10.1901/jaba.2000.33-353

de Souza, D.G., \& de Rose, J.C. (2006). Desenvolvendo programas individualizados para ensino de leitura. Acta Comportamentalia, 14, 77-98.

da Hora, C. L., \& Benvenuti, M. F. L. (2007). Controle restrito em uma tarefa de matching-to-sample com palavras e sílabas: avaliação do desempenho de uma criança diagnosticada com autismo. Revista Brasileira de Análise do Comportamento, 3, 29-45. http://dx.doi.org/10.18542/ rebac.v3i1.822
Dube, W. V., \& Mcllvane, W. J. (1999). Reduction of stimulus overselectivity with nonverbal differential observing responses. Journal of Applied Behavior Analysis, 32, 25-33. http://dx.doi.org/10.1901/jaba.1999.32-25

Dube, W. V., McDonald, S. J., Mcllvane, W. J., \& Mackay, H. A. (1991). Constructed-response matching to sample and spelling instruction. Journal of Applied Behavior Analysis, 24, 305-317. http://dx.doi.org/10.1901/jaba.1991.24-305

Dunn, L. M., \& Dunn, L. M. (1997). Examiner's Manual for the PPVT-III: Peabody Picture Vocabulary Test Third Edition. Circle Pines: American Guidance Service.

Graham, S. (1983). Effective spelling instruction. The Elementary School Journal, 83, 560-567. http://dx.doi.org/ 10.1086/461334

Green, G. (2001). Behavior analytic instruction for learners with autism: Advances in stimulus control technology. Focus on Autism and Other Developmental Disabilities, 16, 72-85. http://dx.doi.org/10.1177/108835760101600203

Hanna, E. S., Karino, C. A., Araújo, V. T., \& de Souza, D. G. (2010). Leitura recombinativa de pseudopalavras impressas em pseudoalfabeto: Similaridade entre palavras e extensão da unidade ensinada. Psicologia USP, 21, 275-311. http://dx.doi.org/10.1590/S010365642010000200005

Mackay, H. A. (1985). Stimulus equivalence reading and in rudimentry spelling. Analysis and Intervention in Developmntal Disabilities, 5, 373-387.

Mackay, H. A., \& Sidman, M. (1984). Teaching new behavior via equivalence relations. In: P. H. Brooks; R. Sperber \& C. McCauley (Eds.), Learning and Cognition in the Mentally Retarded (pp. 493-513). Hillsdale: Erlbaum.

Mackay, H. A., \& Fields, L. (2009). Sintax, grammatical transformation and productivity: A synthesis of stimulus sequences, equivalence classes and contextual control. In: R. A. Rehfeldt \& Y. Barnes-Holmes (Eds.). Derived Relational Responding Applications for Learners with Autism and Other Developmental Disabilities: A progressive guide to change (p. 209-235). Oakland: ContextPress/ New Harbinger Publications.

Paixão, G. M., \& Assis, G. J. A. (2017). Uso do procedimento de Constructed Response Matching to Sample: Uma revisão da literatura. Perspectivas em Análise do Comportamento, 8, 47-60, http://dx.doi.org/10.18761/ PAC.2016.038

Paixão, G. M., de Souza, G. F., Kato, O. M., \& Haydu, V. B. (2013). Análise dos procedimentos de ensino e a emergência da leitura recombinativa. Psicologia da Educação, 36, 5-17.

Pereira, A. M. (2007). Autismo infantil: Tradução e Validação da CARS (Childhood Autism Rating Scale) para Uso no Brasil (Dissertação de mestrado não publicada). Universidade Federal do Rio Grande do Sul, Porto Alegre RS. 


\section{-4: INTERACÃO EM IT PSICOLOGIA}

Perez, W. F., \& Tomanari, G. Y. (2014). Indução de controles por seleção e por rejeição em tarefas de emparelhamento com o modelo: uma revisão metodológica. Acta Comportamentalia, 22, 227-242.

Reis, T. S., Postalli, L. M., \& de Souza, D. G. (2013). Teaching spelling as a route for reading and writing. Psychology \& Neuroscience, 6, 365-373. http://dx.doi.org/10.3922/ j.psns.2013.3.14

Shogren, K., Lang, R., Machalicek, W., Rispoli, M.J., \& O'Reilly, M. (2011). Self-versus teacher management of behavior for elementary school students with asperger syndrome: impact on classroom behavior. Journal of Positive Behavior Interventions, 13, 87-96. http://dx.doi.org/ $10.1177 / 1098300710384508$

Sidman, M., \& Tailby, W. (1982). Conditional discrimination vs. matching to sample: an expansion of the testing paradigm. Journal of the Experimental Analysis of Behavior, 37, 5-22. http://dx.doi.org/10.1901/jeab. 1982.37-5

Spradlin, J. E., \& Brady, N. C. (1999). Early childhood autism and stimulus control. In Ghezzi, P. M., Williams, W. L., \& Carr, J. E. Autism: behavior-analytic perspectives (pp. 4965). New York: Context Press.

Stromer, R., \& Mackay, H. A. (1992). Delayed constructedresponse identity matching improves the spelling performance of students with mental retardation. Journal of Behavioral Education, 2 139-156. http://dx.doi.org/ 10.1007/BF00947117

Stromer, R., Mackay, H. A., Howell, S. R., McVay, A. A., \& Flusser, D. (1996). Teaching computer-based spelling to individuals with developmental and hearing disabilities: transfer of stimulus control to writing tasks. Journal of Applied Behavior Analysis, 29, 25-42. http://dx.doi.org/ 10.1901/jaba.1996.29-25

Tanji, T., \& Noro, F. (2011). Matrix training for generative spelling in children with autism spectrum disorder. Behavioral Interventions, 26, 326-339. http://dx.doi.org/ 10.1002/bin.340

Walpole, C. W., Roscoe, E. M., \& Dube, W. V. (2007). Use of a differential observing response to expand restricted
Glenda Miranda da Paixão e Grauben José Alves de Assis

stimulus control. Journal of Applied Behavior Analysis, 40, 707-712. http://dx.doi.org/10.1901/jaba.2007.707-712

Yamamoto, J., \& Miya, T. (1999). Acquisition and transfer of sentence construction in autistic students: analysis by computer-based teaching. Research in Developmental Retardation, 20(5), 355-377. http://dx.doi.org/10.1016/ S0891-4222(99)00017-7

Data de submissão: 21/03/2017 Primeira decisão editorial: 07/08/2017 Aprovação: 20/11/2017

\footnotetext{
${ }^{1}$ Esvanecimento: transformações graduais de estímulo que visam produzir novas relações ambiente-comportamento, com a minimização da frequência de erros no decorrer do treino (Alves, Assis, Kato e Brino, 2011)
} 